\title{
Millisecond Timescale Synchrony among Hippocampal Neurons
}

\author{
Kamran Diba, ${ }^{1,4}$ Asohan Amarasingham, ${ }^{2,4,5}$ Kenji Mizuseki, ${ }^{3,4,6}$ and György Buzsáki ${ }^{4,6}$ \\ ${ }^{1}$ Department of Psychology, University of Wisconsin-Milwaukee, Milwaukee, Wisconsin 53201, ${ }^{2}$ Department of Mathematics, City College, City University \\ of New York, New York, New York 10031, ${ }^{3}$ Allen Institute for Brain Science, Seattle, Washington 98103, ${ }^{4}$ Center for Molecular and Behavioral Neuroscience, \\ Rutgers University-Newark, Newark, New Jersey 07102, 5Departments of Biology and Psychology, Graduate Center, City University of New York, New York, \\ New York 10031, and ${ }^{6}$ Neuroscience Institute, New York University, New York, New York 10016
}

Inhibitory neurons in cortical circuits play critical roles in composing spike timing and oscillatory patterns in neuronal activity. These roles in turn require coherent activation of interneurons at different timescales. To investigate how the local circuitry provides for these activities, we applied resampled cross-correlation analyses to large-scale recordings of neuronal populations in the cornu ammonis 1 (CA1) and CA3 regions of the hippocampus of freely moving rats. Significant counts in the cross-correlation of cell pairs, relative to jittered surrogate spike-trains, allowed us to identify the effective couplings between neurons in CA1 and CA3 hippocampal regions on the timescale of milliseconds. In addition to putative excitatory and inhibitory monosynaptic connections, we uncovered prominent millisecond timescale synchrony between cell pairs, observed as peaks in the central $0 \mathrm{~ms}$ bin of cross-correlograms. This millisecond timescale synchrony appeared to be independent of network state, excitatory input, and $\gamma$ oscillations. Moreover, it was frequently observed between cells of differing putative interneuronal type, arguing against gap junctions as the sole underlying source. Our observations corroborate recent in vitro findings suggesting that inhibition alone is sufficient to synchronize interneurons at such fast timescales. Moreover, we show that this synchronous spiking may cause stronger inhibition and rebound spiking in target neurons, pointing toward a potential function for millisecond synchrony of interneurons in shaping and affecting timing in pyramidal populations within and downstream from the circuit.

Key words: fast oscillations; gap junctions; hippocampus; interneurons; networks; synchrony

\section{Introduction}

Networks of neurons in the brain generate an assortment of oscillatory patterns. In the hippocampus, prominent oscillations have been noted at $\theta(5-10 \mathrm{~Hz})$, slow $\gamma(30-50 \mathrm{~Hz})$, fast $\gamma(50-90$ $\mathrm{Hz}), \varepsilon(90-140 \mathrm{~Hz})$, and ripple $(130-230 \mathrm{~Hz})$ frequencies (Belluscio et al., 2012). Although numerous studies point to the role of synaptic and nonsynaptic coupling of neurons in the generation of oscillations, specific mechanisms that support the various rhythms are not well understood (Buzsáki and Draguhn, 2004; Klausberger and Somogyi, 2008; Isaacson and Scanziani, 2011). A critical first challenge is determining the nature of neuronal connectivity of in vivo networks. Neurons can communicate with each other either synaptically, electrically through gap junctions,

Received March 18, 2014; revised Sept. 19, 2014; accepted Sept. 24, 2014.

Author contributions: K.D. and G.B. designed research; K.D. and K.M. performed research; A.A. contributed analytic tools; K.D. analyzed data; K.D. and G.B. wrote the paper, with suggestions from all authors.

This work was supported by the University of Wisconsin-Milwaukee Research Growth Initiative to K.D., National Institutes of Health Grant MH102840 to A.A., Japan Society for the Promotion of Science's Research Fellowship for Research Abroad to K.M., and National Institutes of Health Grants MH054671, NS034994, NS074015, and NSF SBE 0542013 to G.B. We thank Anton Sirota, Shigeyoshi Fujisawa, David Sullivan, Eva Pastalkova, Anne Cei, and Michael Zugaro for assistance during preliminary stages of this project.

The authors declare no competing financial interests.

Correspondence should be addressed to Dr. Kamran Diba, Department of Psychology, University of WisconsinMilwaukee, Milwaukee, WI 53201.E-mail: diba@uwm.edu.

DOI:10.1523/JNEUROSCI.1091-14.2014

Copyright $\odot 2014$ the authors $\quad 0270-6474 / 14 / 3414984-11 \$ 15.00 / 0$ or both (Tamás et al., 1998; Galarreta and Hestrin, 1999; Gibson et al., 1999; Fukuda and Kosaka, 2000; Thomson et al., 2002). Electrical coupling has been observed chiefly among neurons of the same interneuron class (Galarreta and Hestrin, 1999; Gibson et al., 1999; Tamás et al., 2000; Deans et al., 2001; Szabadics et al., 2001; Beierlein et al., 2003; Blatow et al., 2003; Galarreta et al., 2004; Mancilla et al., 2007; Fanselow et al., 2008) and has been postulated to promote fast spike synchronization (Traub et al., 2001; Gibson et al., 2005; Hughes and Crunelli, 2007; Mancilla et al., 2007). A recent in vitro study, however, suggested that millisecond synchrony between inhibitory interneurons can be equally well achieved by chemical synapses (Hu et al., 2011).

There are no established methods to discriminate synaptically or electrically connected neurons in extracellular recordings. Presumably, connections between pairs of neurons impact the structure of the spike-trains of connected neurons. Interactions of spiking neurons are most visible in the relatively increased likelihood of a putative target cell to fire immediately following a spike in the reference cell. Consequently, short-latency narrow peaks in the cross-correlograms (CCGs) of neuronal pairs are interpreted as indicative of monosynaptic connections (Perkel et al., 1967; Csicsvari et al., 1998; Barthó et al., 2004; Fujisawa et al., 2008). The recent development of nonparametric spike jitter techniques has further provided a means to test the statistical significance of peaks and valleys in the CCG with respect to a null hypothesis of 


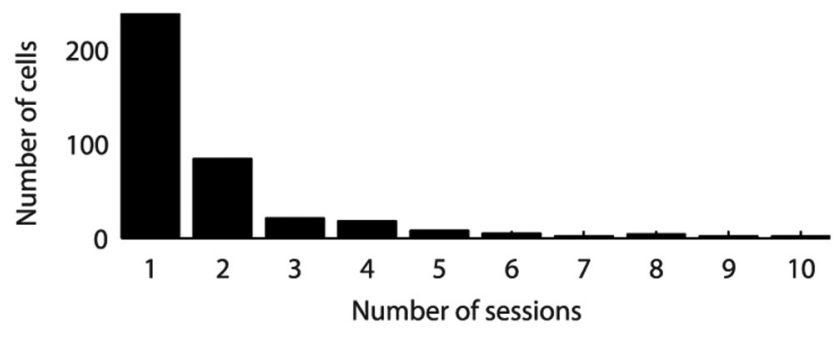

Figure 1. Number of recording sessions per interneuron. Where possible, we tracked interneurons across recording sessions based on spike waveform (for specific examples, see Fig. 7). Tracking was performed very conservatively; most cells could only be reliably attributed to a single session.

fast timescale interactions (Fujisawa et al., 2008; Amarasingham et al., 2012). Here, we apply these techniques to study the full variety of extant connections within large-scale recordings of spiking pyramidal cells and interneurons in the hippocampus of behaving rats.

\section{Materials and Methods}

Data collection and processing. We performed extracellular recordings of large populations of neurons in freely moving male Long-Evans rats, using previously described methods (Diba and Buzsáki, 2008). This involved implanting custom-made drives with 32 and 64 channel silicon probe electrodes (Neuronexus) into the hippocampus of rats under isoflurane anesthesia. After recovery from surgery, water-restricted rats ran on a linear track and were rewarded with water at platforms on the ends of the track. Data were pooled from 7 animals across 80 sessions. Interneurons and pyramidal cells were manually identified based on criterion of firing rate, waveform, burstiness, and refractory period (Csicsvari et al., 1998; Barthó et al., 2004; Sirota et al., 2008) and verified against spatial specificity for pyramidal cells and the lack of spatial specificity for interneurons during activity in the linear track environment. Although imperfect, these criteria are generally considered to be reliable (Csicsvari et al., 1999; Sirota et al., 2008; Mizuseki et al., 2009). Based on the projected waveforms on silicon probe electrodes and experimenter changes to electrode depth, when possible, interneuron single units were tracked over multiple sessions and days, to minimize overcounting of the same cells (Fig. 1).

Pairwise connections. To determine putative monosynaptic connections, as a first "screening" step, all possible pairwise CCGs were calculated with 1 ms size bins and convolved with a 5 ms Gaussian "pseudo-jitter" window (Stark and Abeles, 2009). Pairs with both of their largest waveforms projected onto the same electrode were ignored. Any pairs with $<2.5$ spike counts per CCG bin within $\pm 12 \mathrm{~ms}$ were also discarded from further analysis. For excitatory or millisecond synchronous connections, significant peaks within $3 \mathrm{~ms}$ time lag were noted based on a Poisson approximation and $p$ value $=0.05$. For putative inhibitory connections, we performed the same procedure, but for a significant trough within $3 \mathrm{~ms}$, and further required that a neighboring bin also exhibit a significant trough at $p=0.1$, to account for the slower time course of inhibitory action. This procedure produced a reduced dataset of pairs of interest. To assess significance nonparametrically on this subset, we performed 1000 $5 \mathrm{~ms}$ jitters and $10001 \mathrm{~ms}$ jitters on the spike train of the nonreference cells and calculated global and local 5\% significance bands for CCGs of cell pairs for both jitter timescales (Fujisawa et al., 2008; Amarasingham et al., 2012). Local significance bands were assessed by comparing counts in each bin to surrogate data, whereas global significance bands were assessed by comparing to counts in all bins $\pm 12 \mathrm{~ms}$ (Fujisawa et al., 2008). Global significances were used to determine connections in all subsequent analyses, whereas local significances are used for illustrative purposes. CCGs of cell pairs from the reduced dataset that exhibited a significant peak (relative to $1 \mathrm{~ms}$ jitter) between 1 and $3 \mathrm{~ms}$ were categorized as putative excitatory connections. CCGs of cell pairs from the reduced dataset that exhibited a significant trough (relative to $5 \mathrm{~ms}$ jitter) between 1 and 3 ms were categorized as putative inhibitory connections. CCGs of cell pairs from the reduced dataset that exhibited a significant peak at $0 \mathrm{~ms}$ (relative to $1 \mathrm{~ms}$ jitter) were categorized as millisecond synchronous pairs. Following Agmon (2012), a modified jitter-based synchrony index (JBSI) was calculated for significant millisecond synchronous pairs using real jitter (rather than the virtual jitter implemented by Agmon). If $\tau_{S}=$ bin size ( $1 \mathrm{~ms}$ ), and $\tau_{J}$ is the jitter timescale (1 and $5 \mathrm{~ms}$ in our analysis), JBSI is defined as follows:

$$
J B S I=\beta \frac{N-\left\langle N^{J}\right\rangle}{n_{1}},
$$

where $N$ is the observed number of counts in the peak bin, $\left\langle N^{\top}\right\rangle$ is the number of counts averaged over all jittered surrogate sets, $\beta$ is a normalization constant $=\tau_{I} /\left(\tau_{I}-\tau_{S}\right)$ if $\tau_{I} / \tau_{S}>2$ and $\beta=2$ if $\tau_{J} / \tau_{S} \leq 2$, and $n_{1}$ is the spike count in the lower firing cell in the pair. This measure is useful for comparing the strength of synchrony across cell pairs. A larger JBSI corresponds to a larger peak in the $0 \mathrm{~ms}$ bin relative to baseline firing.

For some analyses, spikes from the reference neuron were further segregated into subsets according to criteria, such as $\gamma$ phase, network state, or the animal's position, to determine how pairwise CCGs depend on these respective criteria. For computational efficiency, we applied the convolution method (Stark and Abeles, 2009) with 5 ms smoothing windows to assess the significances of zero time-lag peaks in these subsets. We note that this method assesses significance based on local, rather than global, significance bands at the jitter timescale.

$\theta$ and $\gamma$ analysis. For each session, the electrode with maximal ripple power was selected for cornu ammonis 1 (CA1) $\theta$ calculations, whereas one electrode with maximal power between 25 and $90 \mathrm{~Hz}$ was selected for subsequent $\gamma$ detection on each shank, to ensure that the oscillation was local. To determine CA3 $\gamma$ phase for CA1 units, we used the electrode corresponding to the first CA3 shank. The $\theta(5-10 \mathrm{~Hz}) / \delta(1-4 \mathrm{~Hz})$ ratio of the power spectral density was calculated using multitaper methods and the Chronux toolbox for MATLAB (MathWorks) (Bokil et al., 2010) in $1 \mathrm{~s}$ windows. To detect state transitions between high and low $\theta$ states, we used custom-made software written by Sirota et al. (2008), which implemented a hidden Markov model (Ghahramani, 2001) using the Hidden Markov Model Toolbox for MATLAB (Kevin Murphy). For each cell, $\theta$ and $\gamma$ phase histograms were determined using the Hilbert transform of the bandpass filtered signal, $5-12 \mathrm{~Hz}$ for $\theta$ and $25-90 \mathrm{~Hz}$ for $\gamma$ (Belluscio et al., 2012). Fitting the data to von Mises distributions provided preferred $\theta$ and $\gamma$ phases.

Ripple detection. One electrode with the maximum average power in the ripple frequency band $(130-230 \mathrm{~Hz})$ during non- $\theta$ states was selected per probe shank in CA1. The signal on each of these selected electrodes was bandpass filtered $(130-230 \mathrm{~Hz})$ and rectified. The following detection algorithm was applied during non- $\theta$ epochs: if the rectified signal exceeded 5 SDs above the mean, ripple detection was triggered. The previous time point at which the signal exceeded 1 SD from the mean was considered the onset of the ripple while the next time point at which the signal dropped $<1 \mathrm{SD}$ from the mean was considered to be the offset of the ripple. Additionally, ripples $<30 \mathrm{~ms}$, or $>450 \mathrm{~ms}$, were excluded, and ripples with inter-ripple intervals $<50 \mathrm{~ms}$ were merged.

Ripple-based classification of interneurons. Following Klausberger et al. (2003), time before and after each detected ripple was normalized, such that -1 indicates the ripple onset, 0 indicates the ripple peak, and 1 indicates the ripple offset. The probability of spiking during ripples was determined for each normalized time bin. When the same interneuron was identified in multiple sessions, ripple data from these sessions were pooled. Based on the resulting periripple time histogram, interneurons were manually assigned to one of nine putative classes (see Fig. 8). Despite some unavoidable ambiguity in such manual sorting, automated $k$-means clustering (MATLAB) produced qualitatively similar results. Subtle category distinctions were clearly subjective (e.g., between Class 1 and 2 and between Class 4 and 5, etc.), but larger distinctions were nevertheless unmistakable (e.g., between Class 1 and 5).

We also tried several other classification schemes (including with automated methods using principal component analysis and $k$-means clustering) based on firing rates, $\theta / \gamma$ phase preferences, and depth of modulation, and spike waveforms and waveform asymmetries. However, we failed to arrive at more satisfactory discriminators. 

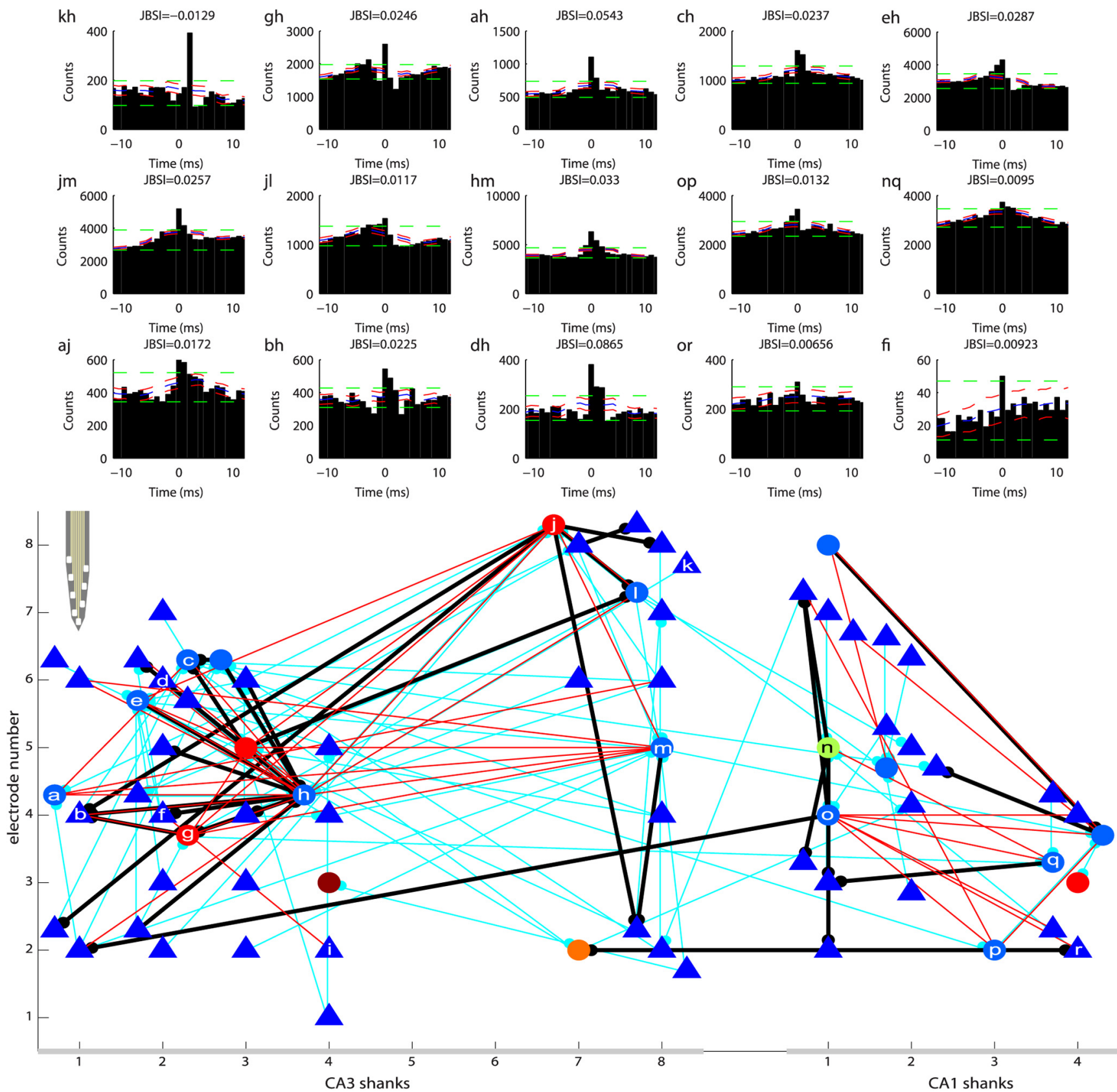

Figure 2. Hippocampal microcircuits in the freely moving rat. Bottom, Network of pyramidal cells (triangles) and interneurons (circles) connected through excitatory (cyan), inhibitory (black), and millisecond synchronous connections (red) in a recording session with an 8 shank $\times 8$ electrode (inset; $\sim 20 \mu \mathrm{m}$ between electrodes, and $200 \mu \mathrm{m}$ between shanks; $x$-axis, shank number) in CA3 and 4 shank $\times 8$ electrodes in CA1. Only connected cells are shown, and distances between cells are not to scale. Letters indicate individual cells. Top, CCGs for pairs comprised of a reference cell (first letter) and target cell (second letter). Connections were assessed based on $95 \%$ global bands using $5 \mathrm{~ms}$ jitter for inhibition, and $1 \mathrm{~ms}$ jitter for excitation and millisecond synchronicity. However, for simplicity, significance bands in all (top) 15 panels are illustrated with $5 \mathrm{~ms}$ jitter. Dashed green line indicates global significance bands; dashed red line indicates local significance bands; blue represents the mean. A sample excitatory connection between pyramidal cell $(\mathrm{k})$ and interneuron $(\mathrm{h})$ is depicted in panel kh. The pair in panel gh exhibit both an inhibitory connection, from cell ( $\mathrm{g}$ ) onto cell (h), as well as millisecond synchronicity, evidenced by a significant peak in the $0 \mathrm{~ms}$ time bin. Additional examples of millisecond synchronous pairs of interneurons are shown in panels ah-nq. An example of a nonsignificant pair (aj) is also shown. For this pair, the CCG peak did not reach significance using $1 \mathrm{~ms} \mathrm{jitter,} \mathrm{although} \mathrm{it} \mathrm{did} \mathrm{with} 5 \mathrm{~ms}$ jitter as shown (see Materials and Methods). Millisecond synchronicity was also observed between pyramidal cells and interneurons both in CA3 (examples bh and dh) and in CA1 (example or). Many such pairs also exhibited an inhibitory connection. In a few rare instances, millisecond synchronicity was observed in pyramidal-pyramidal pairs (fi). JBSI, reflecting the height of the observed central bin relative to jittered spike train, is provided on the top of each panel. Colors of interneuron cell bodies correspond to putative interneuron types defined in Figure 8.

\section{Results}

Significant peaks and troughs in the short-time CCGs of cell pairs were used to infer connectivity between simultaneously recorded neurons in the CA3 and CA1 regions. A sample such network, from a single recording session featuring 67 pyramidal cells and 21 putative interneurons, is illustrated in Figure 2. Here, a total of
76 putative monosynaptic excitatory connections were detected (Fig. 2, cyan). A typical example is provided Figure 2 (kh). In this pair, the probability of spiking increases in cell h $2 \mathrm{~ms}$ after spiking in cell $\mathrm{k}$, indicating that the reference neuron excites the postsynaptic neuron at monosynaptic latency (Barthó et al., 2004). Thirty-seven of 67 pyramidal cells in this session (and 546 of 2398 

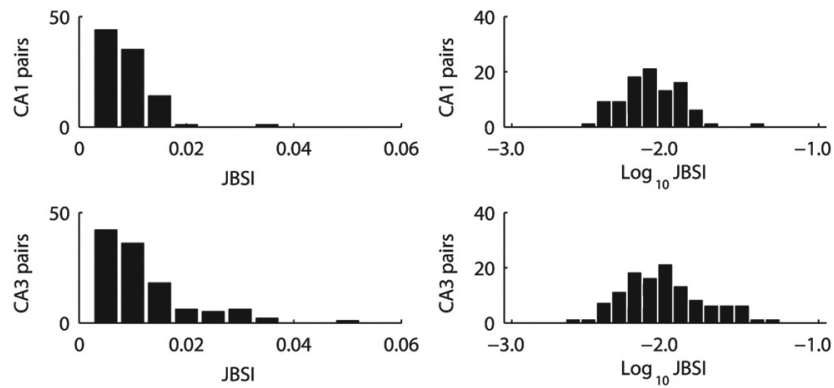

Figure 3. JBSIs for synchronous interneuron pairs in CA3 and CA1. Higher millisecond synchrony is observed among interneuron pairs in CA3 (bottom, median $=0.0083$ ) than in CA1 (top, median $=0.0078$ ), with more CA3 pairs found in the right tail of the skewed distribution (JBSI > 0.02). The same data are shown in both linear (left) and logarithmic (right) scale (Buzsáki and Mizuseki, 2014).
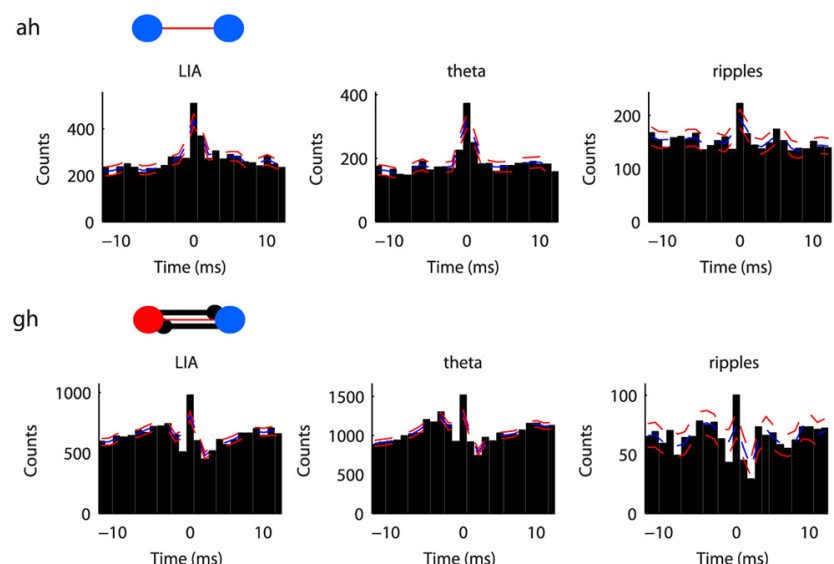

Figure 4. Millisecond synchrony was not dependent on the network state. Significant 0 ms peaks were observed even when spikes were partitioned according to network state, during non- $\theta \mathrm{LIA}, \theta$, and ripples alone (sample pairs ah and gh from Fig. 2). Local significance bands (dashed red line) from $5 \mathrm{~ms}$ jitter are shown, along with the jittered mean (dashed blue line). Similar results were observed in other pairs.

recorded pyramidal cells in all 80 sessions from 7 rats) exhibited at least one excitatory connection, therefore confirming the reference neuron as a pyramidal cell. Thirteen of 21 putative interneurons (and 172 of 688 total interneurons in all sessions in all rats) also exhibited at least one inhibitory connection, with 31 putative monosynaptic inhibitory connections in total, therefore confirming the reference neuron as an inhibitory cell. Several such examples are provided in Figure 2 (eh, gh, and jl). In these instances, decreased probability of spiking followed in the secondary cell $2 \mathrm{~ms}$ after spiking in the reference cell.

Surprisingly, we also observed a considerable fraction of millisecond timescale synchronous connections between cells, as assessed by significantly high counts in the $0 \mathrm{~ms}$ time bin. Forty-one millisecond synchronous connections were observed in the network in Figure 2. Twenty-six of these were between pairs of interneurons (of 210 possible interneuron pairs). Multiple examples of such connections can be seen in Figure 2 (gh through nq): significantly high counts were observed in the $0 \mathrm{~ms}$ bin of these CCGs, indicating that the two cells cofired within $0.5 \mathrm{~ms}$ of each other. The synchronicity in the Figure 2 gh pair in particular takes place despite an overlying antiphasic pattern resulting from mutual inhibition. In this session, a further 15 such zero time lag synchronous connections were between pyramidal cells and interneurons (e.g., Fig. 2, bh, dh, and or), of 1428 possible pyra- ah
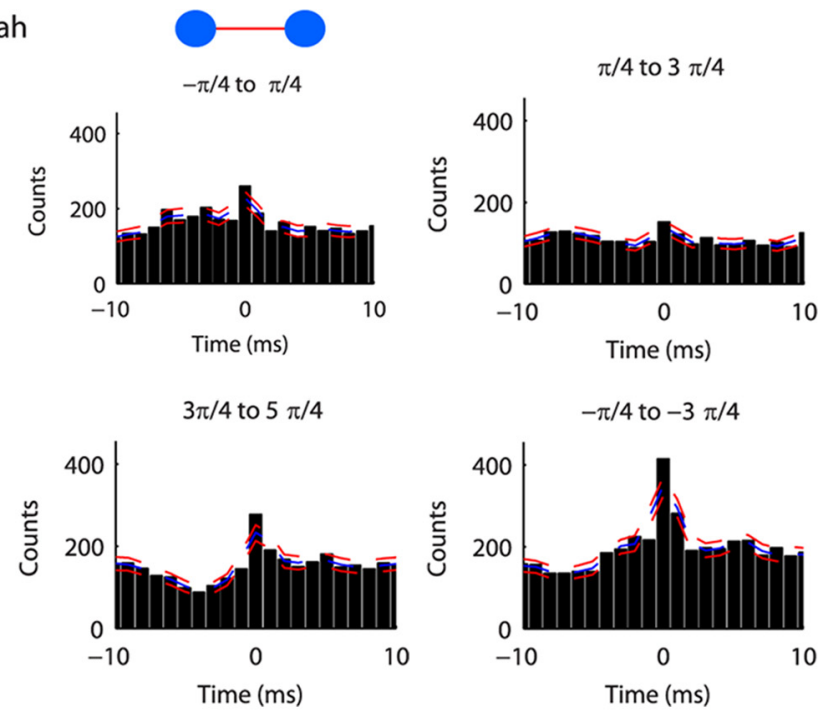

gh
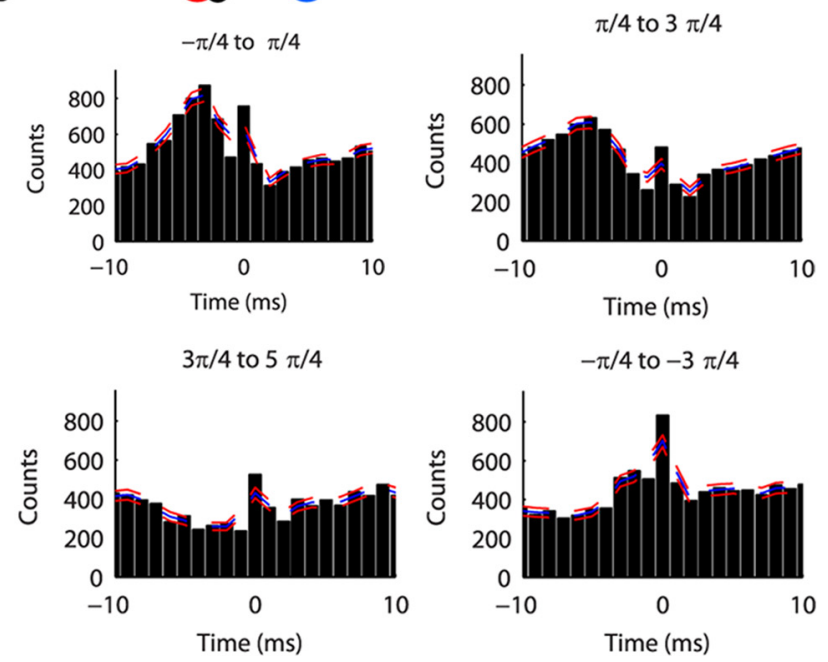

Figure 5. $\quad \gamma$ independence of millisecond synchrony. Significant bin counts were observed at $0 \mathrm{~ms}$ for synchronous pairs when the CCGs were recalculated for subsets of spikes, according to the phase of the local $\gamma$ oscillation recorded from the same shank as the reference neuron. Pairs ah and gh from Figure 2 are illustrated. Four phase quadrants correspond to the rising, peak, descending, and trough phases of $\gamma$. Although $\gamma$ modulation is evident in the CCGs, significant peaks were observed in the $0 \mathrm{~ms}$ bin for all phase quadrants. Similar results were observed in other pairs.

midal-interneuron pairs. In addition, 2 putative pyramidalpyramidal synchronous pairs were observed of 2278 possible such pairs. One example is shown in Figure 2 (fi). Although these pyramidal-pyramidal synchronous pairs were quite rare, it is important to keep in mind that most pyramidal cell pairs do not have overlapping place fields. Consequently, most pyramidal cells do not cofire within $150 \mathrm{~ms}$; and when they do, their firing is often offset by $\theta$ phase precession and the so-called "sequence compression index" (Skaggs et al., 1996; Dragoi and Buzsáki, 2006; Diba and Buzsáki, 2008). With these caveats in mind, detection of any millisecond synchronous pyramidal-pyramidal pairs might still be surprising (see also Takahashi and Sakurai, 2009).

Over all recorded sessions, involving some overlapping sets of recorded neurons, 357 of 3077 interneuron-interneuron pairs 


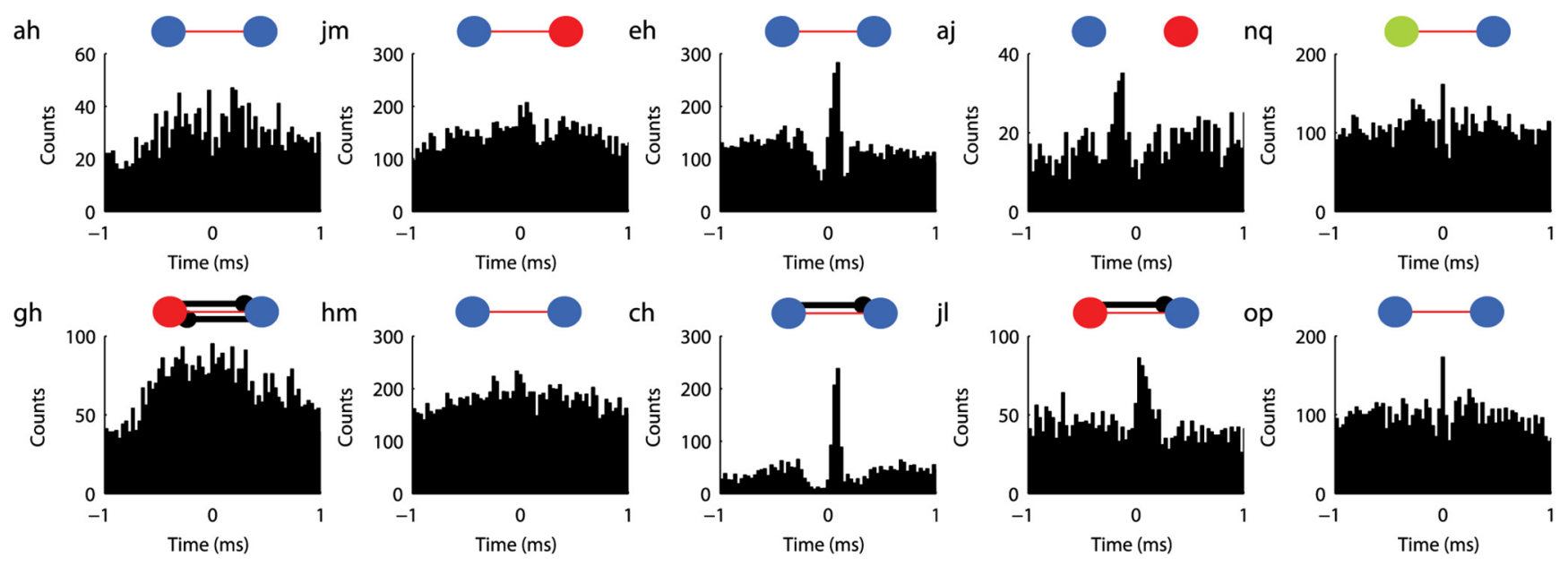

Figure 6. High-resolution cross-correlations between millisecond synchronous pairs. CCGs were calculated with time bins set at the sampling resolution of the recording system (here $0.03 \mathrm{ms)}$. This generally resulted in less obvious peaks between $-0.5 \mathrm{~ms}$ and $0.5 \mathrm{~ms}$, as $0 \mathrm{~ms}$ bin counts were distributed among more (finer) bins (e.g., ah, gh), in which some very small peaks could occasionally be seen (e.g., jm, and hm). However, in a few cases, a very well-defined temporal relationship could be resolved in the spike times of interneuron pairs (e.g., eh, ch), including apparent inhibitory influence surrounding a prominent peak slightly offset from 0 . In other pairs (e.g., aj, jl), wider yet unmistakable peaks were apparent, without the visible troughs. In some pairs, a small and narrow central peak was observable, bordered by negative dips (e.g., nq and op).

$(11.6 \%), 89$ of 22,664 pyramidal-interneuron pairs $(0.39 \%)$, and 60 of 42,062 possible pyramidal-pyramidal pairs $(0.14 \%)$ exhibited millisecond timescale synchrony. In contrast, in six surrogate datasets where we first randomly jittered the entire spike train by $5 \mathrm{~ms}$ and then repeated all of the analyses, we observed only $\{M=6.0 ; 4 \leq x \leq 9\}$ interneuroninterneuron pairs, $\{M=13.8 ; 9 \leq x \leq 20\}$ pyramidal-interneuron pairs, and $\{M=$ 5.8; $2 \leq x \leq 11\}$ pyramidal-pyramidal pairs, indicating that these phenomena were extremely unlikely to have been produced by chance (e.g., assuming that the conditioned likelihood on observed interactions is Gaussian, a two sample $t$ test gives $p=10^{-10}, p=2 \times 10^{-5}, p=4 \times$ $10^{-5}$, respectively). Comparison of CA3 and CA1 neurons across these recordings revealed millisecond synchrony for significantly more CA3 interneuron pairs (227 of $979,23.2 \%)$ than CA1 interneuron pairs $\left(129\right.$ of $893,14.5 \% ; \chi^{2}(1, N=$ 1872) $\left.=23.17, p=10^{-6}\right)$, indicating greater millisecond synchrony between interneuron cell pairs in CA3 than in CA1. Some of these pairs involved the same neurons recorded in multiple sessions. Among unique significant pairs (95 in CA1 and 116 in CA3), some 0 ms peaks in CA3 CCGs appeared more prominent than those in CA1 (e.g., Fig. 2, ah and ch vs op and nq). To quantify this observation, we used a JBSI introduced by Agmon (2012) based on the ratio of the observed counts in the $0 \mathrm{~ms}$ bin to the firing rate of the slower firing neuron in the pair. As seen in Figure 3, CA3 interneuron pairs ( median $=0.0093$ ) showed a more positively skewed distribution of JBSIs ( $p=0.0129$, one-sided two-sample Kolmogoro$\mathrm{v}-$ Smirnov test) than did CA1 interneuron pairs (median $=$ 8 recording electrodes.

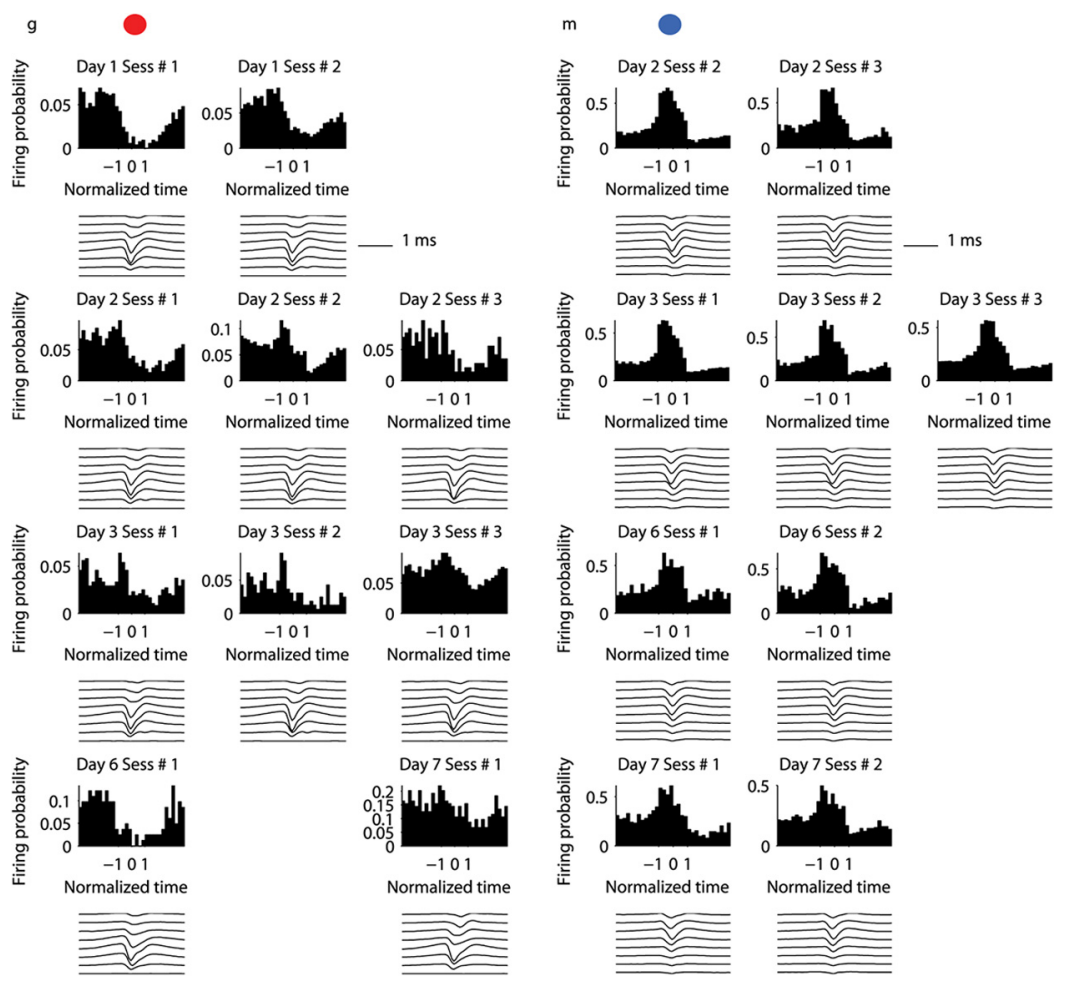

Figure 7. Interneuron firing responses during sharp-wave ripples were generally consistent across days. Cells $g$ and $m$ from Figure 2 are shown across multiple recording days and sessions. Multiple sessions from different recording days are depicted. Top panel for each session, Probability of firing averaged across all ripples detected during that session. Time was normalized into 32 bins, with 0 corresponding to the peak power in the ripple frequency band $(130-230 \mathrm{~Hz})$, and ripple onset and offset corresponding to -1 and 1 , respectively. Bottom panel for each session, Average waveform of spikes attributed to the interneuron on each of

$0.0078)$ and had significantly more JBSIs $>0.02$ (16 vs $1 ; \chi^{2}(1$, $N=17)=11.44, p=0.0008)$.

Next, we examined whether such connections were statedependent. We divided spikes into three groups according to the oscillatory state of the network: $\theta$, non- $\theta$, or large-amplitude irregular activity (LIA) (Vanderwolf, 1969) and ripples. Prominent peaks continued to be observed for each of the three network 
a
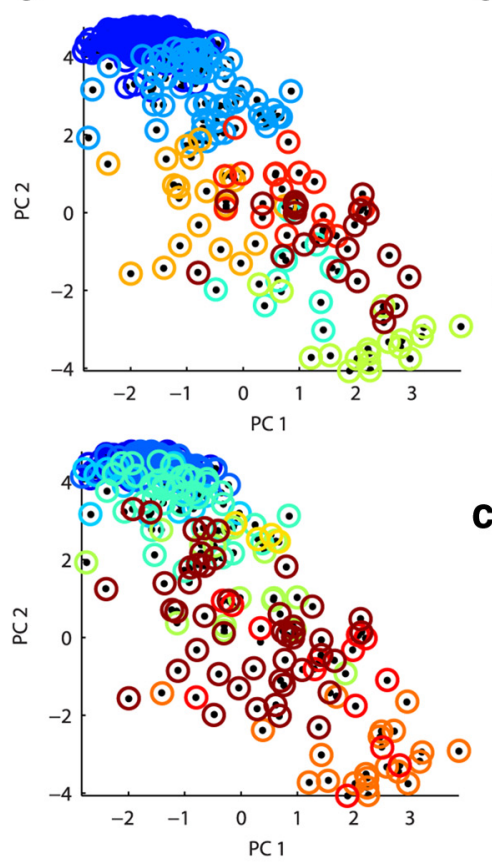

b
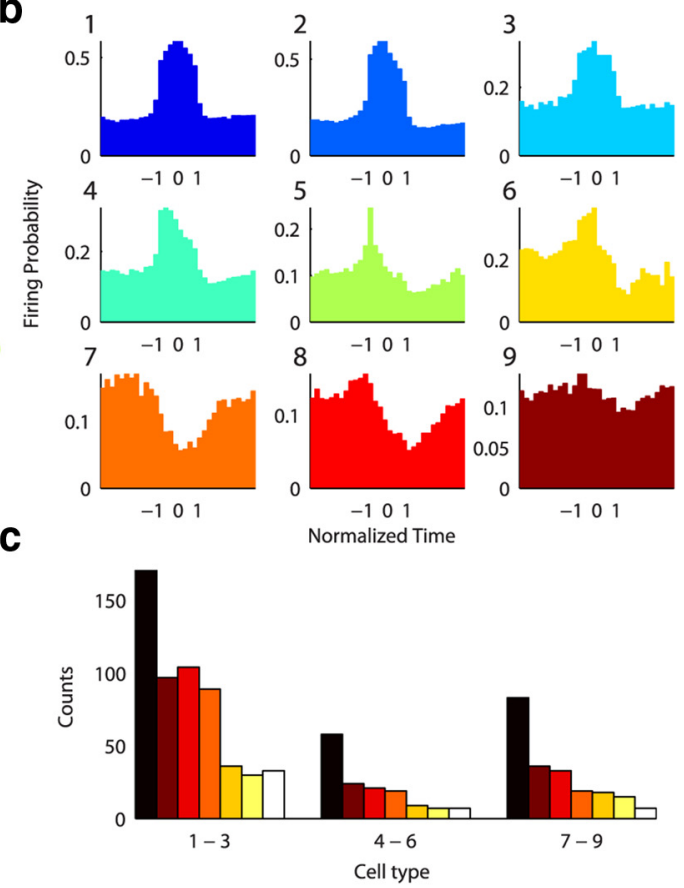

Figure 8. Putative classification of interneurons based on firing response during sharp-wave ripples. Interneurons were sorted into nine classes (shown in different colors) based on visual inspection of firing during sharp-wave ripples averaged across all connectable sessions (Fig. 7). Although some of the sorting was inevitably subjective, classification approximately corresponded to $k$-means clusters obtained from principal component features from the time-normalized firing responses to ripples from all interneurons recorded in sessions with at least 20 ripples. $\boldsymbol{a}$, Top, Manual clusters with corresponding colors to $\boldsymbol{b}$, with nearby classes chosen to be close in color space. Bottom, Automated clusters based on top five principal components (arbitrary coloring) along first and second principal components. $\boldsymbol{b}$, The ripple firing response for all cells assigned to each class of nine classes were averaged. Normalized time -1 indicates the ripple onset, 0 indicates the ripple peak, and 1 indicates the ripple offset. Overall, some cells firing robustly during ripples (blue Group 1-3), whereas others showed transient and varying responses (green Group 4-6) or decreased in firing (red Group 7-8) or failed to show a discernible response (9). The colors used for interneurons in Figure 2 were based on the same color/category relationships. $c$, Histograms for these cell types display the distribution of the total number of cells in all recordings with at least 20 ripples that were assigned to each class group (black), those that were excited by a simultaneously recorded pyramidal unit (brown), those that displayed millisecond synchrony (red), those that displayed millisecond synchrony with cells from Classes 1-3 (orange), those that displayed millisecond synchrony with cells from Classes 4-9 (dark yellow), those that inhibited at least one other simultaneously recorded cell (light yellow), and those that were inhibited by a simultaneously recorded cell (white). These color codes are unique to this panel and not used elsewhere. Synchronous pairs were observed in all nine cell types (data not shown), and all cell types formed synchronous pairs with cells in the blue group (Types 1-3).

states. Examples from pairs ah and gh of Figure 2 are illustrated in Figure 4. Overall, of 212 synchronous pairs, significant $0 \mathrm{~ms}$ peaks were observed in all 212 pairs during $\theta$ alone, and in 211 pairs during LIA alone, as assessed by the convolved CCG method with $5 \mathrm{~ms}$ Gaussian pseudo-jitter (Stark and Abeles, 2009).

Principal neurons in the hippocampus fire in a locationspecific manner (O'Keefe and Dostrovsky, 1971; McNaughton et al., 1996) and comprise the main excitatory input of interneurons. To test whether common input from populations of place cells might account for the observed millisecond timescale synchrony in interneuron pairs, for each significant pair we split the spike train of the reference neuron according to the animal's position, with an equal number of spikes in each of three nonoverlapping spatial sectors, and retested for significant $0 \mathrm{~ms}$ peaks using $5 \mathrm{~ms}$ Gaussian pseudo-jitter (Stark and Abeles, 2009). Because different place cells are active in each of these spatial sectors (Diba and Buzsáki, 2008), we reasoned that excitatory inputs to interneurons would also be different for each sector. Despite the reduced statistical power resulting from splitting up the spike train, in 182 of 212 pairs $(85.9 \%)$, the $0 \mathrm{~ms}$ peaks were significant in all three spatial sectors. This suggests that common input from place cells alone cannot fully account for all of the observed millisecond synchrony.

To establish that millisecond timescale synchrony did not arise from comodulation by the $\gamma$ rhythm, all $0 \mathrm{~ms}$ time bin peaks were evaluated from global significance bands using very conservative $1 \mathrm{~ms}$ jitters of the secondary spike train. Consequently, rejection of the null hypothesis in these pairs means that mechanisms operating at timescales slower than $1 \mathrm{~ms}$ (frequency $<1000 \mathrm{~Hz}$ ) do not account for these observations (Amarasingham et al., 2012). Jitter of $1 \mathrm{~ms}$ likely results in an underestimate of the number of synchronous pairs; for example, pair aj in Figure 2 did not reach significance at $1 \mathrm{~ms}$ jitter (but did so at $5 \mathrm{~ms}$ as shown). Nevertheless, as a secondary control, we divided spikes of reference neurons into four quadrants based on the $\gamma$ phase of the spike (Fig. 5) and recalculated CCGs and tested significance with the convolution method with 5 ms Gaussian pseudo-jitter (Stark and Abeles, 2009). Again, this reduction of sample size naturally reduces statistical power, increasing the Type II (false negative) error rate, so we do not expect all subsets to retain significant peaks. Yet, although the $\gamma$ oscillation clearly modulated the cross-correlations between cell pairs, as evident by comparing the CCGs in Figure 5 at the trough of $\gamma$ $(-\pi / 4$ to $\pi / 4)$ with those from the $\gamma$ peak ( $3 \pi / 4$ to $5 \pi / 4)$, 195 of $212(92.0 \%)$ millisecond synchronous cell pairs continued to demonstrate significant $0 \mathrm{~ms}$ peaks in at least 3 of 4 four $\gamma$ phase quartiles (and 148 of $212,69.8 \%$ cell pairs maintained significant $0 \mathrm{~ms}$ peaks in all four quartiles).

Although our hypothesis testing and corresponding statistical power were based on 1-ms-wide bins, our recordings were sampled at a higher temporal resolution (20 and $32.552 \mathrm{kHz}$ ). Therefore, we asked whether resolving the pairwise cross-correlations with a finer time bin would better elucidate the nature of the millisecond interactions (Fig. 6). We retested for peaks in the finer CCG between $-0.5 \mathrm{~ms} 0.5 \mathrm{~ms}$ for all pairs that displayed $0 \mathrm{~ms}$ synchrony at $5 \mathrm{~ms}$ jitter, now using single sample time bins $(0.03 \mathrm{~ms}$ and $0.05 \mathrm{~ms}$ ). For most synchronous pairs (59.4\%), the higher resolution of the cross-correlation did not reveal any notable features (Fig. 6, ah, jm, gh, hm). However, in a subset of these cases $(4.7 \%)$, the relative spiking timing in cell pairs revealed exquisite temporal interactions on a much finer timescale (Fig. 6, eh and ch). In such pairs, the CCGs indicated an ultra-fast synchronizing action lasting on the order of $0.1 \mathrm{~ms}$ bordered by prominent troughs. Other pairs also revealed noticeable but wider off-central (Fig. 6, aj and jl) peaks (10.4\%). Such timing relationships seem to be inconsistent with common excitatory input to these neurons as the underlying source of the synchrony but may reflect the propagation of spikes through gap junctions. A few showed some small subtle peaks (14.6\%; Fig. 6, jm and $\mathrm{hm})$, and yet other pairs (10.8\%) demonstrated narrow $0.03 \mathrm{~ms}$ 
central peaks (Fig. 6, nq and op), flanked by similar timescale dips in the CCG. The majority (20 of 23) of pairs with the latter type peaks were CA1-CA1 interneuron pairs. The diversity of submillisecond timescale interactions observed suggests a possible range of mechanisms underlying millisecond timescale synchrony in hippocampal networks.

Gap junctions between synchronous interneurons are a potential source for the millisecond timescale synchrony. The majority of gap junctions have been reported between cells of the same interneuron class in the cortex (Gibson et al., 1999), although some gap junctions have been observed between cells of different classes as well (Gibson et al., 1999, 2005; Beierlein et al., 2000; Venance et al., 2000). In our experiments, it was not possible to definitively identify the neuronal class of recorded neurons. However, juxtacellular recordings from CA1 and CA3 neurons in urethaneanesthetized rats have indicated that, during hippocampal network oscillations, neurons of the same class display similar spiking patterns (Klausberger and Somogyi, 2008). The spiking responses during ripples in particular varied most distinctly between different neuronal types with, for instance, the firing of parvalbumin (PV)-positive basket cells increasing robustly and symmetrically, that of bistratified cells also increasing but asymmetrically so, then decreasing over the ripple duration, axoaxonic cells transiently firing at the onset but then silent for the remainder of the ripple, and oriens-lacunosum-moleculare cells silent during the duration of ripples (Klausberger et al., 2003, 2004, 2005; Klausberger and Somogyi, 2008). In a subset of our recordings, we were able to track individual cells over multiple sessions across multiple days (Fig. 7). We observed some variability in the ripple responses of these interneurons across sessions, indicating that some apparent ripple differences may arise from signal detection and variability across recording sessions. Nevertheless, there were also some consistent aspects to the responses to ripples; for example, during ripples, cell g fired very little and was inhibited for at least some portion of the ripple, whereas cell $\mathrm{m}$ consistently increased firing (Fig. 7). Thus, broader aspects of the ripple responses might indeed correspond to consistent features of different interneurons.

Motivated by this classification scheme in the anesthetized preparation, we evaluated the firing response during ripples for interneurons in our drug-free dataset. A variety of responses were observed, which we subjectively categorized into each of nine putative classes (Fig. 8). Although these categorizations were clearly not unambiguous, types farthest away in number and color likely captured some differences. We tested whether these rippledefined classes were different in their preferred phase of firing during CA1 $\theta$ and CA3 $\gamma$ oscillations. The clustering of cells from different classes (Fig. 9) showed a grouping of CA1 interneurons in Classes 7-9 at the rising phase of CA3 $\gamma$ but different phase preferences for other classes. Relative to CA1 $\theta$, CA1 and CA3 interneurons attributed to Classes 1-3 (putative "PV" and "bistratified" cells) (Klausberger et al., 2003, 2004) tended to fire at the opposite $\theta$ phase than did cells of Classes 7-9 (putative "oriens-lacunosum-moleculare" and "cholecystokinin" cells) (Klausberger et al., 2003, 2005). Interestingly, these cell b
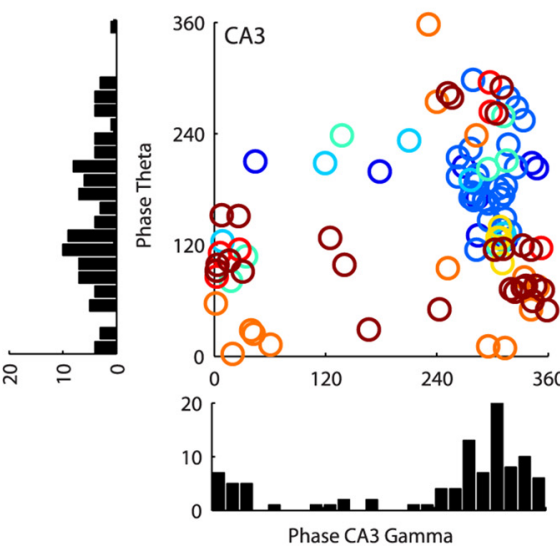

Phase CA3 Gamma

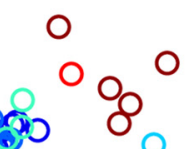

000

CA3 Gamma

were composed based on preferred firing phase of neurons during CA3 $\gamma$ and CA1 $\theta$ phase for cells in both regions. Some general group differences could be observed in the clustering of phase preferences of different neuronal types; for example, Type 4-6 (green) and Type 7-9 (orange) interneurons in CA1 (a) showed different $\theta$ and $\gamma$ phase preferences, and Type 1-3 (blue) and Type 7-9 (orange) classes in CA3 (b) showed different $\theta$ e same as in Figure $8 b$.

bh

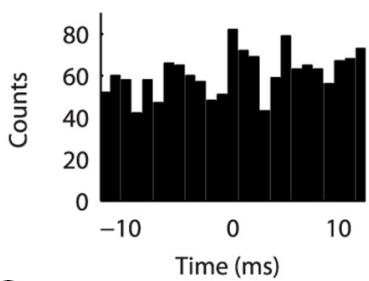

a

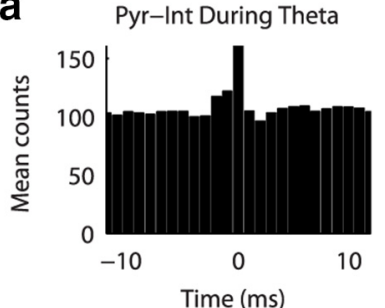

C

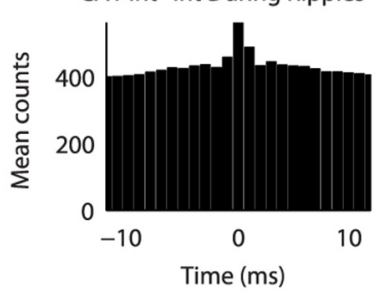

or

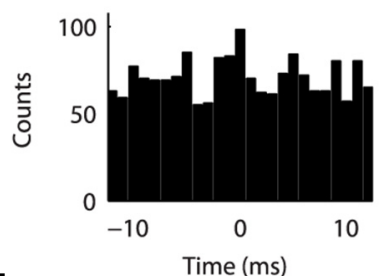

b

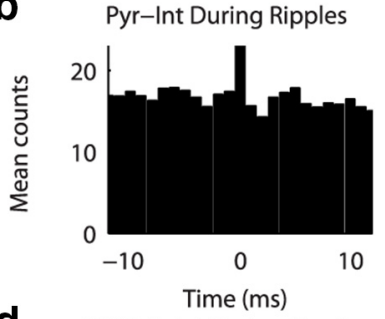

d CA3 Int-Int During Ripples

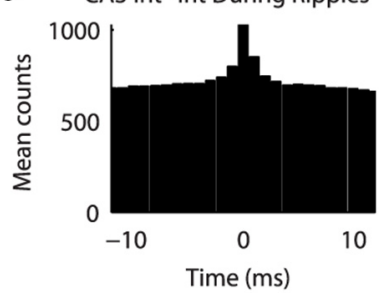

Figure 10. Millisecond timescale synchrony between pyramidal cells and interneurons oscillates during sharp-wave ripples. The CCG of these pairs (Fig. 2, pair bh in CA3 and pair or in CA1) using spikes firing during ripples alone indicates they did not fire antiphasically, as might be expected in interneuron-pyramidal pairs, but instead fired with apparent oscillatory coupling at $\sim 200 \mathrm{~Hz}$, corresponding to the ripple oscillation. Data pooled from significant 0 ms pyramidal-interneuron pairs across all sessions shows millisecond synchrony during $\theta(\boldsymbol{a})$ and during ripples $(\boldsymbol{b})$, with notable secondary peaks at 5 $\mathrm{ms}$ and $-6 \mathrm{~ms}$. Similarly, pooled data from significant $0 \mathrm{~ms}$ interneuron-interneuron pairs in CA3 (c) or in CA1 (d) across all sessions show millisecond synchrony during ripples but without visible secondary peaks.

types show similar opposite firing during ripples (e.g., compare these types in Fig. $8 b$ ), suggesting a consistent antagonism between these types on a timescale on the order of $100 \mathrm{~ms}$ (Varga et al., 2012), possibly mediated by afferent projections from the medial-septum (Dragoi et al., 1999; Borhegyi et al., 2004). 


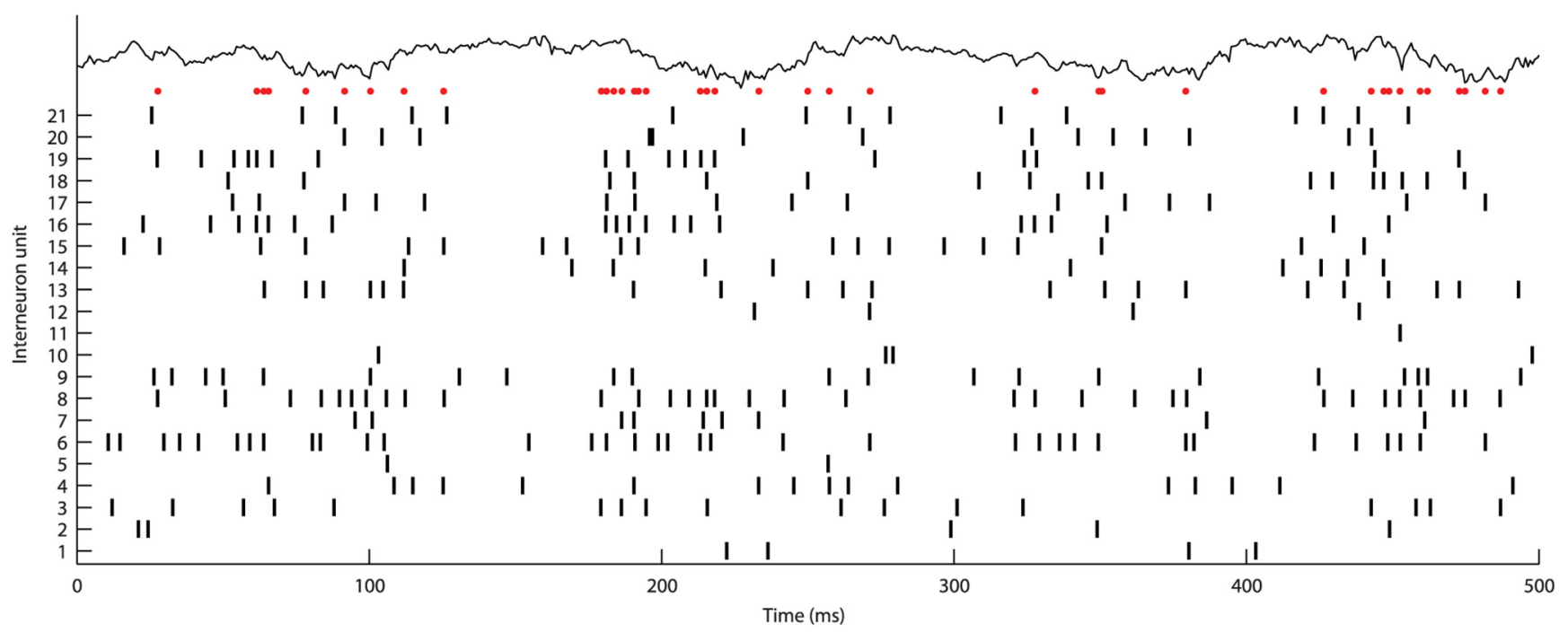

Figure 11. Sample interneuron population raster in a freely moving rat. Shown here is a $500 \mathrm{~ms}$ raster plot from the 21 interneurons depicted in Figure 2 , along with the CA1 pyramidal layer local field potential on the top. Red dots indicate instances of synchronized firing between two or more cells. Millisecond synchronous firing appeared to be largely stochastic and naturally varying with firing rates, with different interneurons synchronizing at different time points.

Using the above novel classification scheme, we tested whether synchronous pairs could arise from cells of different classes. Because membership in cell Classes 1-3 was the least ambiguous and most commonly observed, we tested whether cells belonging to these classes formed millisecond synchronous pairs with cells belonging to other classes. Surprisingly, millisecond synchronicity was consistently observed regardless of interneuron class. Overall, $43.6 \%$ of synchronous cell pairs involved one cell from Types 1-3, and one cell from Types 4-9, $42.7 \%$ of pairs involved two cells both of Types $1-3$, and the remaining $13.7 \%$ of pairs involved two cells both of Types 4-9. Examples of such cross-type synchronicity can also be seen in Figure 2, where millisecond synchronous pairs were frequently observed between red (Type 8 ) and blue (Type 2) cells (e.g., gh, hj, jm, gm, etc.), which fire very differently during ripples, and between a green (Type 5) and blue (Type 2) cell (nq). Millisecond synchronous pairings involved all different interneuron types (Fig. $8 c$ ). Interestingly, as noted above, synchronicity was also observed for some pyramidal cell-interneuron pairs. During sharp-wave ripples in particular, the CCGs of these pairs demonstrated fast oscillatory coupling, with secondary peaks at $\pm 5 \mathrm{~ms}$ (Fig. 10bh-d). Although such pyramidal-interneuron synchronous pairs were rare and so possibly inconsequential, we did not generally see such secondary peaks during ripples in other pairs, including interneuron-interneuron pairs (Fig. 10d), suggesting that this type of synchronous coupling may contribute to faster $\varepsilon$ $(90-140 \mathrm{~Hz})$ and ripple band $(130-230 \mathrm{~Hz})$ oscillations.

We examined whether millisecond timescale synchronicity was brought about by distinct population bursts, but we failed to find reliable signatures of instances in which multiple neurons exhibited simultaneous synchronicity. Instead, synchronous pairs of spikes were observed at seemingly random times (Fig. 11). Thus, rather than arising from a single source of global synchrony, these patterns appear to arise from more subtle interactions between small networks of neurons. Throughout this example session, $39.4 \%$ of spikes by any interneuron fired within $0.5 \mathrm{~ms}$ of spiking by at least one other interneuron on a different electrode, relative to the $37.1 \%$ ( $\pm 0.1 \% \mathrm{SD}$ ) expected by chance ( 1000 surrogates with $5 \mathrm{~ms}$ jitter; $p<0.001$ ). Naturally, this latter chance percentage indicates that even independently firing in- terneurons will cofire by chance based on their firing rates and comodulation by network rhythms. Nevertheless, enhanced millisecond timescale synchrony is a common feature of spiking patterns among interneurons. Over all sessions, the ratio of observed synchronous spikes to nonsynchronous spikes varied according to the dataset, including the number of simultaneously recorded neurons, from $1.1 \%$ to $38.3 \%$ (mean $23.9 \%$ ), within CA3 alone, and 1.8\%-22.1\% (mean $13.0 \%$ ) within CA1 alone.

What function does such fast synchronicity serve? To help answer this question, we examined the effect of millisecond synchronicity on pyramidal cell populations in the CA1 and CA3 regions. We pooled millisecond synchronous spikes from all interneurons (such as the aforementioned $39.4 \%$ of interneuron spikes in the sample session) and compared them with an equal number of nonsynchronous spikes from the same cells (e.g., randomly selected from the complementary $60.6 \%$ of spikes in the sample session) across all sessions. We calculated the CCG of synchronous and nonsynchronous interneuron spikes separately with CA1 and CA3 pyramidal cells (Fig. 12). Synchronous spikes in CA1 (Fig. 12a) were more likely to have been preceded by the firing of CA1 pyramidal cells in the bins $2 \mathrm{~ms}$ before the synchronous interneuron spikes, indicating that strong excitatory input may play a role in providing synchronicity. Consistent with this view, we also observed increased upstream CA3 pyramidal cell firing before CA1 interneuron synchronicity. Importantly, synchronous spikes were more effective at suppressing the firing of CA1 pyramidal cells than were nonsynchronous spikes. A similar effect was also observed for synchronous spiking of CA3 interneurons (Fig. 12b), which were preceded by increased firing, showed more effective inhibition in CA3 than did nonsynchronous spikes, and were followed by a larger excitatory rebound in CA1 pyramidal cells. Thus, millisecond timescale synchrony of interneuron spikes increases the efficacy of their action on pyramidal cell targets in both CA3 and CA1 and may result in a secondary wave of firing in CA1 pyramidal cells.

\section{Discussion}

In the course of evaluating the connectivity within simultaneously recorded networks of neurons in the hippocampus, we made several key observations. First, we observed frequent milli- 
a

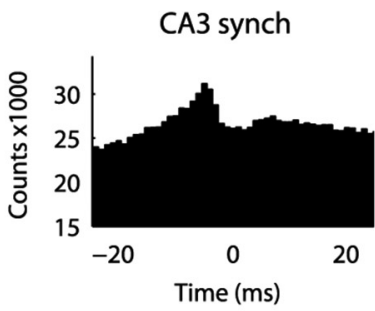

CA3 non-synch

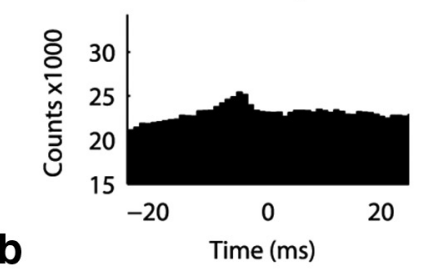

CA3 synch

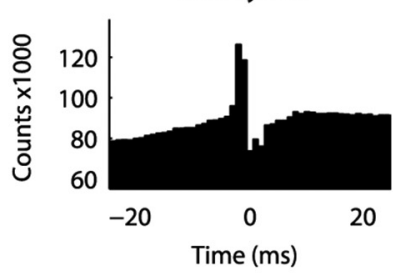

CA3 non-synch
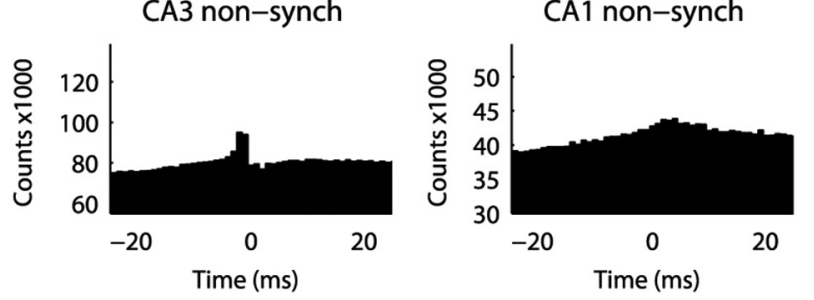

Figure 12. Effect of millisecond synchrony on pyramidal populations. All interneuron spikes across all sessions were labeled according to whether they occurred within $0.5 \mathrm{~ms}$ of spikes of interneurons on other electrodes. For synchronous spiking of CA1-CA1 cell pairs, (a) CCGs were calculated between synchronous interneuron spikes and all CA3 pyramidal cell spikes (top left) and all CA1 pyramidal cell spikes (top right). $y$-axes indicate total spike counts $(\times 1000)$ from all combined pyramidal cell spikes. These can be compared with similar CCGs calculated between an equal number subset of randomly selected nonsynchronous interneuron spikes and pyramidal cell firing in CA3 (bottom left) and CA1 (bottom right). These comparisons demonstrate greater immediate inhibition in CA1 along with a subsequent (postinhibitory) increase in excitation after millisecond synchronous activity. $\boldsymbol{b}$, For synchronous interneuron spiking in CA3CA3 cell pairs, CCGs were calculated between synchronous interneuron spikes and all CA3 pyramidal cell spikes (top left) and all CA1 pyramidal cell spikes (top right). These can be compared with similar CCGs calculated between an equal number of randomly selected nonsynchronous interneuron spikes and pyramidal cell firing in CA3 (bottom left) and CA1 (bottom right). These comparisons demonstrate that CA3 pyramidal cell spiking was more likely to precede, and less likely to follow, synchronous spiking in CA3 interneurons. Furthermore, a stronger subsequent rise in pyramidal cell activity can be seen in CA1 after these events.

second timescale synchronous coordination between interneurons belonging to different putative classes and, occasionally, between pyramidal cells and interneurons. Hypothesis testing based on spike jitter global significance bands showed that the mechanism by which these synchronicity arises operates at timescales faster than $1 \mathrm{~ms}$, rendering $\gamma$ or ripple oscillations unlikely as the underlying source for their genesis (Amarasingham et al., 2012). Furthermore, we observed such synchronous coordination during different network states, including $\theta$ and ripple oscillations, all phases of the $\gamma$ oscillation, as well as during non- $\theta$, no-ripple LIA epochs. Such high temporal precision of interneuron synchrony may arise from multiple mechanisms.

Some interneurons in our network were likely to be connected through gap junctions. Indeed, at high resolution, some pairs of the same putative class demonstrated timing relationships that would appear to be consistent with submillisecond propagation of activity across electrical connections (Deans et al., 2001; Galarreta and Hestrin, 2001). However, several factors suggest that millisecond synchrony does not arise by gap junctions alone. First, we frequently observed millisecond synchrony between interneurons of different putative classes, which are considered to have a low $(<5 \%)$ probability of forming electrical synapses (Hestrin and Galarreta, 2005). Second, we observed millisecond synchrony in pairs that were recorded on shanks $>1 \mathrm{~mm}$ apart, substantially greater than the dendritic arborization in most hippocampal interneurons (Freund and Buzsáki, 1996), making it unlikely that membrane of all of the millisecond synchronous pairs had sufficient overlap to form gap junctions. Third, some in vitro experiments suggest that, through gap junctions, neurons affect the probability of firing in connected neurons mostly 1-10 ms following an action potential (Tamás et al., 2000; Gibson et al., 2005), rather than the $<0.5$ ms reported here.

In our in vivo dataset, we observed that synchronous interneuron spiking was more likely to be preceded by spiking of pyramidal cells within $<2 \mathrm{~ms}$. This appears to indicate that synchronized excitation may play a role in synchronizing interneurons at the millisecond timescale. On the other hand, to produce synchronous firing of two cells in the central $0 \mathrm{~ms}$ bin alone $(<0.5 \mathrm{~ms}$ apart) as observed, a "third-party" excitation mechanism (Brunel and Wang, 2003) would require interneuron pairs to be electrically equidistant from the upstream pyramidal neurons, with matched conduction delays (Izhikevich, 2006), so that the action potential would arrive simultaneously to the interneuron synapses. These conditions may be met for a subset but not all of our interneuron pairs. Furthermore, the membrane of both interneurons would need to be charged to spike at a similar speed. This requirement would also imply that EPSP-spike charge time is similar across several interneuron classes to account for the observed zero time lag synchrony between these interneurons. Such fine-tuned timing appears unlikely to take place in all of the observed synchronous pairs, particularly given the fineresolution (submillisecond) synchrony in some instances (e.g., Fig. 6, eh, ch). Moreover, in synchronous pairs, millisecond synchronicity was not spatially restricted within the maze environment, meaning that common input from a few place cells would be insufficient to produce the effect. Instead, producing spatially independent millisecond synchrony would require precise coordination across a large population of pyramidal cell inputs. Although we cannot rule out that such coordination arises from pyramidal cell axonal gap junctions (Traub et al., 2002), we find this scenario unlikely. Pyramidal-pyramidal millisecond synchronous pairs in our dataset were extremely rare, whereas such synchronicity was readily observable among interneuron pairs. In sum, our data cannot exclude a role for common input but indicate that the common inputs alone cannot fully explain the observed millisecond synchrony.

A potential scenario is that the inhibitory connections themselves play a significant role in providing the conditions appropriate for such widespread coordinated excitation and the subsequent millisecond synchrony, possibly supported by gap junction-mediated activity and common excitatory inputs. Interestingly, Gibson et al. (2005) showed that fast timescale synchrony can occur between cells connected by inhibitory synapses 
alone but that this synchrony becomes more robust in the presence of electrical synapses. Another intriguing mechanism was recently proposed by $\mathrm{Hu}$ et al. (2011). They observed similar millisecond synchronicity in in vitro experiments among PV and non-PV interneurons that appeared to lack electrical connections. Based on intracellular data and biophysical modeling, they proposed that inhibition alone is sufficient to synchronize neurons at such fast timescales, presumably through rebound. However, the precise rebound mechanisms, by which such superfast synchrony can be secured, needs to be elucidated. In particular, postinhibitory rebound appears to be strongly dependent on neuromodulatory tone and network oscillations (Adhikari et al., 2012), whereas the millisecond synchrony in our recordings was robust to the state of the network.

Regardless of the mechanisms, the function supported by such fast synchrony appears to be important. Interneurons may synchronize to create a larger postsynaptic effect (Stark et al., 2013). By increasing the likelihood of coactivity between neuron pairs, this synchronicity may help to support oscillatory coupling at various frequencies (Gibson et al., 2005; Mancilla et al., 2007). Indeed, our findings show that millisecond precision of synchronized interneuron spiking produced a larger postsynaptic inhibitory effect than was achieved by interneurons acting in isolation. Precise timing on this timescale may be relevant for neuronal computation (Yang et al., 2008; Yang and Zador, 2012). The propensity for fast synchronous firing among interneurons has also been suggested to render a network more sensitive to synchronous excitatory input, therefore allowing it to function as an ultra-fast coincidence detector (Galarreta and Hestrin, 2001) and providing an effective means for the propagation of this activity to downstream targets. Consistent with this notion, we observed an increased probability of spiking activity in CA1 pyramidal cells subsequent to synchronous interneuron spiking, relative to nonsynchronous spiking, in both CA3 and CA1 networks. Although an increased upstream excitatory drive may induce millisecond synchrony in parallel with a propagating wave of excitation, overall, our experiments illustrate that millisecond synchronous activity of interneurons can exert a local effect that will also be reflected in downstream targets.

\section{References}

Adhikari MH, Quilichini PP, Roy D, Jirsa V, Bernard C (2012) Brain state dependent postinhibitory rebound in entorhinal cortex interneurons. J Neurosci 32:6501-6510. CrossRef Medline

Agmon A (2012) A novel, jitter-based method for detecting and measuring spike synchrony and quantifying temporal firing precision. Neural Syst Circ 2:5. CrossRef Medline

Amarasingham A, Harrison MT, Hatsopoulos NG, Geman S (2012) Conditional modeling and the jitter method of spike resampling. J Neurophysiol 107:517-531. CrossRef Medline

Barthó P, Hirase H, Monconduit L, Zugaro M, Harris KD, Buzsáki G (2004) Characterization of neocortical principal cells and interneurons by network interactions and extracellular features. J Neurophysiol 92:600-608. CrossRef Medline

Beierlein M, Gibson JR, Connors BW (2000) A network of electrically coupled interneurons drives synchronized inhibition in neocortex. Nat Neurosci 3:904-910. CrossRef Medline

Beierlein M, Gibson JR, Connors BW (2003) Two dynamically distinct inhibitory networks in layer 4 of the neocortex. J Neurophysiol 90:29873000. CrossRef Medline

Belluscio MA, Mizuseki K, Schmidt R, Kempter R, Buzsáki G (2012) Crossfrequency phase-phase coupling between theta and gamma oscillations in the hippocampus. J Neurosci 32:423-435. CrossRef Medline

Blatow M, Rozov A, Katona I, Hormuzdi SG, Meyer AH, Whittington MA, Caputi A, Monyer H (2003) A novel network of multipolar bursting interneurons generates theta frequency oscillations in neocortex. Neuron 38:805-817. CrossRef Medline
Bokil H, Andrews P, Kulkarni JE, Mehta S, Mitra PP (2010) Chronux: a platform for analyzing neural signals. J Neurosci Methods 192:146-151. CrossRef Medline

Borhegyi Z, Varga V, Szilágyi N, Fabo D, Freund TF (2004) Phase segregation of medial septal GABAergic neurons during hippocampal theta activity. J Neurosci 24:8470-8479. CrossRef Medline

Brunel N, Wang XJ (2003) What determines the frequency of fast network oscillations with irregular neural discharges? I. Synaptic dynamics and excitation-inhibition balance. J Neurophysiol 90:415-430. CrossRef Medline

Buzsáki G, Draguhn A (2004) Neuronal oscillations in cortical networks. Science 304:1926-1929. CrossRef Medline

Buzsáki G, Mizuseki K (2014) The log-dynamic brain: how skewed distributions affect network operations. Nat Rev Neurosci 15:264-278. CrossRef Medline

Csicsvari J, Hirase H, Czurko A, Buzsáki G (1998) Reliability and state dependence of pyramidal cell-interneuron synapses in the hippocampus: an ensemble approach in the behaving rat. Neuron 21:179-189. CrossRef Medline

Csicsvari J, Hirase H, Czurkó A, Mamiya A, Buzsáki G (1999) Oscillatory coupling of hippocampal pyramidal cells and interneurons in the behaving rat. J Neurosci 19:274-287. Medline

Deans MR, Gibson JR, Sellitto C, Connors BW, Paul DL (2001) Synchronous activity of inhibitory networks in neocortex requires electrical synapses containing connexin36. Neuron 31:477-485. CrossRef Medline

Diba K, Buzsáki G (2008) Hippocampal network dynamics constrain the time lag between pyramidal cells across modified environments. J Neurosci 28:13448-13456. CrossRef Medline

Dragoi G, Buzsáki G (2006) Temporal encoding of place sequences by hippocampal cell assemblies. Neuron 50:145-157. CrossRef Medline

Dragoi G, Carpi D, Recce M, Csicsvari J, Buzsáki G (1999) Interactions between hippocampus and medial septum during sharp waves and theta oscillation in the behaving rat. J Neurosci 19:6191-6199. Medline

Fanselow EE, Richardson KA, Connors BW (2008) Selective, statedependent activation of somatostatin-expressing inhibitory interneurons in mouse neocortex. J Neurophysiol 100:2640-2652. CrossRef Medline

Freund TF, Buzsáki G (1996) Interneurons of the hippocampus. Hippocampus 6:347-470. Medline

Fujisawa S, Amarasingham A, Harrison MT, Buzsáki G (2008) Behaviordependent short-term assembly dynamics in the medial prefrontal cortex. Nat Neurosci 11:823-833. CrossRef Medline

Fukuda T, Kosaka T (2000) The dual network of GABAergic interneurons linked by both chemical and electrical synapses: a possible infrastructure of the cerebral cortex. Neurosci Res 38:123-130. CrossRef Medline

Galarreta M, Hestrin S (1999) A network of fast-spiking cells in the neocortex connected by electrical synapses. Nature 402:72-75. CrossRef Medline

Galarreta M, Hestrin S (2001) Spike transmission and synchrony detection in networks of GABAergic interneurons. Science 292:2295-2299. CrossRef Medline

Galarreta M, Erdélyi F, Szabó G, Hestrin S (2004) Electrical coupling among irregular-spiking GABAergic interneurons expressing cannabinoid receptors. J Neurosci 24:9770-9778. CrossRef Medline

Ghahramani Z (2001) An introduction to hidden Markov models and Bayesian networks. Int J Pattern Recognition Artificial Intelligence 15:9-42. CrossRef

Gibson JR, Beierlein M, Connors BW (1999) Two networks of electrically coupled inhibitory neurons in neocortex. Nature 402:75-79. CrossRef Medline

Gibson JR, Beierlein M, Connors BW (2005) Functional properties of electrical synapses between inhibitory interneurons of neocortical layer 4 . J Neurophysiol 93:467-480. CrossRef Medline

Hestrin S, Galarreta M (2005) Electrical synapses define networks of neocortical GABAergic neurons. Trends Neurosci 28:304-309. CrossRef Medline

Hu H, Ma Y, Agmon A (2011) Submillisecond firing synchrony between different subtypes of cortical interneurons connected chemically but not electrically. J Neurosci 31:3351-3361. CrossRef Medline

Hughes SW, Crunelli V (2007) Just a phase they're going through: the complex interaction of intrinsic high-threshold bursting and gap junctions in the generation of thalamic alpha and theta rhythms. Int J Psychophysiol 64:3-17. CrossRef Medline

Isaacson JS, Scanziani M (2011) How inhibition shapes cortical activity. Neuron 72:231-243. CrossRef Medline 
Izhikevich EM (2006) Polychronization: computation with spikes. Neural Comput 18:245-282. CrossRef Medline

Klausberger T, Somogyi P (2008) Neuronal diversity and temporal dynamics: the unity of hippocampal circuit operations. Science 321:53-57. CrossRef Medline

Klausberger T, Magill PJ, Márton LF, Roberts JD, Cobden PM, Buzsáki G, Somogyi P (2003) Brain-state- and cell-type-specific firing of hippocampal interneurons in vivo. Nature 421:844-848. CrossRef Medline

Klausberger T, Márton LF, Baude A, Roberts JD, Magill PJ, Somogyi P (2004) Spike timing of dendrite-targeting bistratified cells during hippocampal network oscillations in vivo. Nat Neurosci 7:41-47. CrossRef Medline

Klausberger T, Marton LF, O’Neill J, Huck JH, Dalezios Y, Fuentealba P, Suen WY, Papp E, Kaneko T, Watanabe M, Csicsvari J, Somogyi P (2005) Complementary roles of cholecystokinin- and parvalbumin-expressing GABAergic neurons in hippocampal network oscillations. J Neurosci 25: 9782-9793. CrossRef Medline

Mancilla JG, Lewis TJ, Pinto DJ, Rinzel J, Connors BW (2007) Synchronization of electrically coupled pairs of inhibitory interneurons in neocortex. J Neurosci 27:2058-2073. CrossRef Medline

McNaughton BL, Barnes CA, Gerrard JL, Gothard K, Jung MW, Knierim JJ, Kudrimoti H, Qin Y, Skaggs WE, Suster M, Weaver KL (1996) Deciphering the hippocampal polyglot: the hippocampus as a path integration system. J Exp Biol 199:173-185. Medline

Mizuseki K, Sirota A, Pastalkova E, Buzsáki G (2009) Theta oscillations provide temporal windows for local circuit computation in the entorhinalhippocampal loop. Neuron 64:267-280. CrossRef Medline

O'Keefe J, Dostrovsky J (1971) The hippocampus as a spatial map: preliminary evidence from unit activity in the freely-moving rat. Brain Res 34 : 171-175. CrossRef Medline

Perkel DH, Gerstein GL, Moore GP (1967) Neuronal spike trains and stochastic point processes: II. Simultaneous spike trains. Biophys J 7:419440. CrossRef Medline

Sirota A, Montgomery S, Fujisawa S, Isomura Y, Zugaro M, Buzsáki G (2008) Entrainment of neocortical neurons and gamma oscillations by the hippocampal theta rhythm. Neuron 60:683-697. CrossRef Medline

Skaggs WE, McNaughton BL, Wilson MA, Barnes CA (1996) Theta phase precession in hippocampal neuronal populations and the compression of temporal sequences. Hippocampus 6:149-172. CrossRef Medline

Stark E, Abeles M (2009) Unbiased estimation of precise temporal correlations between spike trains. J Neurosci Methods 179:90-100. CrossRef Medline

Stark E, Eichler R, Roux L, Fujisawa S, Rotstein HG, Buzsáki G (2013)
Inhibition-induced theta resonance in cortical circuits. Neuron 80:12631276. CrossRef Medline

Szabadics J, Lorincz A, Tamás G (2001) Beta and gamma frequency synchronization by dendritic GABAergic synapses and gap junctions in a network of cortical interneurons. J Neurosci 21:5824-5831. Medline

Takahashi S, Sakurai Y (2009) Sub-millisecond firing synchrony of closely neighboring pyramidal neurons in hippocampal CA1 of rats during delayed non-matching to sample task. Front Neural Circuits 3:9. CrossRef Medline

Tamás G, Somogyi P, Buhl EH (1998) Differentially interconnected networks of GABAergic interneurons in the visual cortex of the cat. J Neurosci 18:4255-4270. Medline

Tamás G, Buhl EH, Lörincz A, Somogyi P (2000) Proximally targeted GABAergic synapses and gap junctions synchronize cortical interneurons. Nat Neurosci 3:366-371. CrossRef Medline

Thomson AM, West DC, Wang Y, Bannister AP (2002) Synaptic connections and small circuits involving excitatory and inhibitory neurons in layers $2-5$ of adult rat and cat neocortex: triple intracellular recordings and biocytin labelling in vitro. Cereb Cortex 12:936-953. CrossRef Medline

Traub RD, Kopell N, Bibbig A, Buhl EH, LeBeau FE, Whittington MA (2001) Gap junctions between interneuron dendrites can enhance synchrony of gamma oscillations in distributed networks. J Neurosci 21:9478-9486. Medline

Traub RD, Draguhn A, Whittington MA, Baldeweg T, Bibbig A, Buhl EH, Schmitz D (2002) Axonal gap junctions between principal neurons: a novel source of network oscillations, and perhaps epileptogenesis. Rev Neurosci 13:1-30. CrossRef Medline

Vanderwolf $\mathrm{CH}$ (1969) Hippocampal electrical activity and voluntary movement in the rat. Electroencephalogr Clin Neurophysiol 26:407-418. CrossRef Medline

Varga C, Golshani P, Soltesz I (2012) Frequency-invariant temporal ordering of interneuronal discharges during hippocampal oscillations in awake mice. Proc Natl Acad Sci U S A 109:E2726-E2734. CrossRef Medline

Venance L, Rozov A, Blatow M, Burnashev N, Feldmeyer D, Monyer H (2000) Connexin expression in electrically coupled postnatal rat brain neurons. Proc Natl Acad Sci U S A 97:10260-10265. CrossRef Medline

Yang Y, Zador AM (2012) Differences in sensitivity to neural timing among cortical areas. J Neurosci 32:15142-15147. CrossRef Medline

Yang Y, DeWeese MR, Otazu GH, Zador AM (2008) Millisecond-scale differences in neural activity in auditory cortex can drive decisions. Nat Neurosci 11:1262-1263. CrossRef Medline 